\title{
Channel Allocation Based on Content Characteristics for Video Transmission in Time-Domain-Based Multichannel Cognitive Radio Networks
}

\author{
Md. Jalil Piran, M. Ejaz Ahmed, Amjad Ali, Ju Bin Song, and Doug Young Suh \\ Electronics and Radio Engineering, Yongin-si 446-701, Republic of Korea \\ Correspondence should be addressed to Doug Young Suh; suh@khu.ac.kr
}

Received 27 July 2015; Revised 18 August 2015; Accepted 19 August 2015

Academic Editor: Yuh-Shyan Chen

Copyright (C) 2015 Md. Jalil Piran et al. This is an open access article distributed under the Creative Commons Attribution License, which permits unrestricted use, distribution, and reproduction in any medium, provided the original work is properly cited.

\begin{abstract}
This paper proposes a method for channel allocation based on video content requirements and the quality of the available channels in cognitive radio networks (CRNs). Our objective is to save network bandwidth and achieve high-quality video delivery. In this method, the content is divided into clusters based on scene complexity and PSNR. To allocate channel to the clusters over multichannel CRNs, we first need to identify the licensee's activity and then maximize the opportunistic usage accordingly. Therefore, we classify short and long time transmission opportunities based on the licensee's activities using a Bayesian nonparametric inference model. Furthermore, to prevent transmission interruption, we consider the underlay mode for transmission of the clusters with a lower bitrate. Next, we map the available spectrum opportunities to the content clusters according to both the quality of the channels and the requirements of the clusters. Then, a distortion optimization model is constructed according to the network transmission mechanism. Finally, to maximize the average quality of the delivered video, an optimization problem is defined to determine the best bitrate for each cluster by maximizing the sum of the logarithms of the frame rates. Our extensive simulation results prove the superior performance of the proposed method in terms of spectrum efficiency and the quality of delivered video.
\end{abstract}

\section{Introduction}

Spectrum crowding is drastically increasing as the great evolution of mobile and multimedia communication services drives demand for higher bitrates. The inefficient spectrum utilization of traditional fixed spectrum allocation motivated authorities to allow opportunistic spectrum access to licensed spectrum bands by unlicensed users in CRNs. These secondary users (SUs), without explicit negotiation, autonomously sense the bands and opportunistically try to capture the interleaved portions by tuning their operating parameters without interfering with the primary users (PUs). Thus, the licensed channel idle time distribution is specific to PU activity.

Improved spectrum utilization is a positive development for high-volume video communication. Video transmission is loss tolerant and rate adaptive, whereas in data transmission each bit is compulsory [1]. The problem of video transmission over CRNs is complicated by heterogeneity in the application contents as well as network fluctuations. In this paper, we propose a video transmission scheme for CRNs that allocates time domain spectral opportunities to achieve high-quality video delivery while saving network resources.

Although many schemes for video transmission over CRNs have already been proposed, they did not consider the unique characteristics of the video content. The proposed scheme, called two-tier cooperative spectrum allocation [2], considers SUs' specific QoS requirements as constraint conditions for spectrum allocation and sets targets to achieve the cooptimization of spectrum utilization and SUs' spectrum hand-off rates. Yu et al. [3] proposed an integrated design approach to jointly optimize the application-layer QoS for multimedia transmission over CRNs. Based on sensed channel conditions, SUs adapt an intrarefreshing rate at the application layer in addition to the parameters of other layers. To achieve the best user-perceived real-time video 
quality for SUs of wireless CRNs, a quality-driven cross-layer system for joint optimization of system parameters across the entire network protocol stack is proposed by Luo et al. [4]. The proposed system models time variations of primary network usage and wireless channels to jointly optimize encoder behavior, cognitive MAC scheduling, transmission, and modulation and coding for SUs in a systematic way on a distortion-delay framework to provide the best video quality as perceived by SUs. To improve the performance of real-time video transmission using cross-layer design and a cognitive approach, an extension of the approach proposed by Luo et al. [4] to an optimized multichannel cognitive MAC has been proposed by Thirunavukkarasu et al. [5] considering optimal channel selection based on the channel sensing order described by Cheng and Zhuang [6]. Real-time distributed multiagent learning [7] is a cross-layered framework at different OSI layers based on spectrum utilization and multimedia QoS parameters that dynamically exploits available spectrum bands while using available interference information to obtain learning efficiency. Wang et al. [8] proposed a single-layer QoS approach for delay-sensitive voice service over CRNs based on the time division multiple access technique for wireless local area networks. However, each part of video content can have different characteristics, which means the existing solutions might not be sufficient to satisfy its unique requirements and simultaneously save network resources.

To alleviate the issue, we first cluster the content based on scene complexity and PSNR, where complex and simple scenes have high and low bitrates, respectively. Second, to identify PU activity, we use a Bayesian nonparametric approach to organize the SU cluster parameters to capture the channel idle time distribution and accordingly maximize transmission strategy. A crucial challenge for SUs is estimating the PU idle time distribution. Therefore, statistics on the time domain opportunities for different PU traffic patterns should be considered. We thus concentrate on offering the time domain whitespaces between successive PU packets specific to primary traffic patterns. Hence, short and long time slots are considered as the time between successive primary packet arrivals and the absence of a $\mathrm{PU}$, respectively.

The content clusters with a higher bitrate are thus transmitted over channels with high bitrates and the most reliable modulation and FEC schemes, whereas the clusters with lower bitrates are allocated to channels with lower quality, low modulation, and low FEC schemes. Because adaptive modulation and channel coding are good techniques for using channel fluctuations to maximize content data rate under a known residual BER constraint, we assume several choices of modulation schemes, for example, QPSK, 16-QAM, and 64-QAM, along with some FEC schemes with the rate of $1 / 2$, $2 / 3$, and $3 / 4$. Under the same channel circumstances, various modulation coding schemes result in various data rates and symbol error rates as well. Such techniques let us use the channel variations to maximize the video data rate under a known residual bit error rate constraint. When an appropriate channel is captured by an SU, the user should adopt a modulation coding scheme to support a higher throughput rate. On the other hand, when the available channel is not in a good condition in terms of bitrate, the user should adopt a low-rate modulation scheme. Furthermore, whereas the underlay mode is characterized by low bitrate and low power, the clusters with lower bitrate are an attractive solution at the arrival time of PUs to prevent transmission interruption.

In addition, we define an optimization problem to minimize video distortion by the network transmission mechanism. To achieve the main objective of the paper, we optimized the bitrate of each segment by maximizing the sum of the logarithms of the frame rates based on available network opportunities. We evaluate the performance of the proposed method in terms of video delivery quality and successful video transmission time normalized by the PU idle-busy cycle.

The remainder of the paper is organized as follows. In the next section, the system framework and problem formulation are discussed in detail. Then, simulation settings and results are presented in Section 3. Section 4 concludes the paper.

\section{System Framework}

We use an energy-detection technique to sense PUs' activity because of its efficiency and fast noncoherent features that essentially compute a running average of the signal power over a window of prespecified spectrum length. However, sensing of the PUs is imprecise due to noise and a limited number of observations; the errors are known as the probability of false detection $\left(\operatorname{Pr}_{\text {False-alarm }}\right)[9,10]$. The probability of false alarm and miss detection are recalculated with the help of complete and incomplete gamma and Marcum Qfunctions:

$$
\begin{gathered}
H_{0} \text { : Presence of Signal if }\left(\sum_{n=1}^{N}\left(R_{s}[n]\right)^{2}\right)>\lambda, \\
H_{1} \text { : Absence of Signal if }\left(\sum_{n=1}^{N}\left(R_{s}[n]\right)^{2}\right)<\lambda, \\
\operatorname{Pr}_{\text {False_alarm }}=\operatorname{Pr}\left\{\left(\sum_{n=1}^{N}\left(R_{s}[n]\right)^{2}\right)>\lambda \mid H_{0}\right\} \\
=\frac{\Gamma(m, \lambda / 2)}{\Gamma(m)}, \\
\operatorname{Pr}_{\text {miss }}=1-Q\left(\left(\frac{\lambda}{\delta^{2}}-1-\gamma\right)^{\frac{U N}{1+2 \gamma}}\right) \\
\operatorname{Pr}_{\text {detection }}=e^{-\lambda / 2} \sum_{k=0}^{m-2} \frac{1}{k !}\left(\frac{\lambda}{2}\right)^{2}+\left(\frac{1+\gamma}{\gamma}\right)^{m-1} \\
\cdot\left(e^{\lambda / 2(1+\gamma)}-e^{-\lambda / e} \sum_{k=0}^{m-2}\left(\frac{\lambda \gamma}{2(1+\gamma)}\right)^{k}\right)
\end{gathered}
$$

where $R_{s}$ is the received signal, $N$ is number of samples, $\gamma$ is SNR, $m$ is the Marcum $Q$-function factor, $\delta^{2}$ is the AWGN noise variance, $\lambda$ is the detection threshold, and $U$ is the number of SUs. From (2), it can be observed that 
$\mathrm{P}_{\text {False_alarm }}$ is independent of SNR; hence under $H_{0}$ means the presence of the PU. The probabilities that the PU will remain inactive during the SU transmission or sensing periods are the following equations, respectively:

$$
\begin{aligned}
& \operatorname{Pr}_{t}^{S}=\left(\frac{\zeta^{C}}{\zeta^{C}+\Gamma_{S}}\right)^{\zeta^{C}}, \\
& \operatorname{Pr}_{t}^{T}=\left(\frac{\zeta^{C}}{\zeta^{C}+\Gamma_{T}}\right)^{\zeta^{H}},
\end{aligned}
$$

where $\zeta^{C}, \zeta^{H}$ are scale and shape parameters, respectively. To evaluate the quality of the available channels, SUs exploit the traffic pattern of PUs to optimize their transmissions. The accessible channels are in two modes: short and long time slots. A short time slot is based on PU activity (time opportunities between successive PUs' transmissions), whereas a long time slot is offered to SUs when no PU is inactive. To discern PU activity, we use a Bayesian nonparametric clustering method. We consider packet length $P_{l}$, packet interarrival time $P_{\text {int }}$, and variance in packet length $\Delta$ as the features of PU activity. The packet length for various traffic payloads is different; for example, gaming applications have longer packet size than VoIP packets [11]. Suppose that $X_{n}$ is the feature space vector of PU activity, $X_{i}=\left[P_{l_{i}}, P_{\text {int }_{i}}, \Delta_{i}\right]^{T}$, with matrix representation as $X=\left[x_{1}, x_{2}, \ldots, x_{n}\right]$. The probability density functions of the features are

$$
\begin{gathered}
P\left(P_{l}\right)=\int_{P_{\text {int }}} \int_{\Delta} \vec{\theta} d P_{\text {int }} d \Delta, \\
P\left(P_{\text {int }}\right)=\int_{P_{l}} \int_{\Delta} \vec{\theta} d P_{l} d \Delta, \\
P\left(P_{\Delta}\right)=\int_{P_{\text {int }}} \int_{P_{l}} \vec{\theta} d P_{\text {int }} d p_{l} .
\end{gathered}
$$

The packet arrival model follows a Pareto long-tail distribution from $P\left(P_{\text {int }}\right)$. We use a variational technique to convert the inference problem into an optimization problem as a nonparametric generative model for inference. Because in our framework the PU traffic model is unknown and its variation is based on time, we model the feature space using an instance of the nonparametric Bayesian models. Distribution of $x_{n}$ based on the condition of cluster assignment variable $z_{i}$ and cluster parameters $\theta$ is

$$
\begin{aligned}
p\left(x_{n} \mid z_{n}, \vec{\theta}_{1}, \vec{\theta}_{2}, \ldots\right) \\
=\prod_{i=1}^{\infty} h\left(x_{n}\right) \exp \left\{\vec{\theta}_{i}^{K} x_{n}-\widetilde{\alpha}\left(x_{n}\right)\right\}^{1\left[z_{n=i}\right]},
\end{aligned}
$$

where $\widetilde{\alpha}(\cdot)$ is the appropriate cumulant generating function and $h(\cdot)$ is a nonnegative function. The probability of short time availability at time $t$ is

$$
P_{t, \text { short_opp }}=1-\frac{D\left(\zeta^{H}-1\right)[1-Q(\Delta)]}{\zeta^{C} \zeta^{H}} .
$$

From (5), (6), and (9), the probability that $\mathrm{PU}$ is inactive at time $t$ and remains idle during SU transmission $\Gamma_{T}$ is $\left[P_{t} F_{t}^{T}\right]$, and the probability that the SU transmits a packet successfully with acknowledgment is

$$
P_{t} F_{t}^{T}\left(1-\xi_{0}\right)+\left(1-\xi_{t} F_{t}^{T}\right)\left(1-\xi_{1}\right)
$$

where $\xi_{0}, \xi_{1}$ are probabilities of an unsuccessful SU transmission because of fading and collision with PUs, respectively. The probability of SU successful transmission in a long time slot is

$$
P_{t} F_{t, \text { long_opp }}^{T}\left(1-\xi_{0}\right)+\left(1-P_{t} F_{t, \text { long_opp }}^{T}\right)\left(1-\xi_{1}\right) .
$$

PUs have an exclusive access right to the licensed bands, so to prevent transmission interruption we switch transmission of the clusters with lower bitrate to underlay mode in the presence of a PU. The overlay mode for SUs is expressed in terms of bit error rate performance [12], whereas, in underlay mode, CR transmitted power spectral density is restricted under a predefined interference threshold:

$$
\text { Overlay } P_{\mathrm{err}}=Q\left(\sqrt{\frac{2 E_{b_{s}}}{2 \sum_{k=1}^{k} M_{k} E_{b_{p}} / N_{f}+N_{0}}}\right) \text {, }
$$

Underlay $P_{\text {err }}$

$$
=Q\left(\sqrt{\frac{2}{\left(2 \sum_{k=1}^{k} M_{k} / N_{f}\right)\left(E_{b_{p}} / E_{b_{s}}\right)+N_{0} / E_{b, s}}}\right),
$$

where $\sum_{k=1}^{K} M_{k}$ is the total number of subchannels occupied by PUs, $N_{f}$ is the number of nonoverlap frequency subchannels over the entire bandwidth, $E_{b_{p}}$ and $E_{b_{s}}$ are the bit energy of the PU and SU, respectively, $N_{0}$ is the noise contribution, and $K$ is the total number of PUs. The packet error rate is calculated as $1-(1-P(e))^{\text {packet size }}$. Throughout a transmission, the server maintains the weighted average and standard deviation of the idle and busy duration of the short and long time slots over available channels. Hence, prior to availability of an idle interval, the server prepares segment $S_{i}$ of the content for transmission.

We design a practical streaming scheme that distributes packets over multiple channels according to their available bandwidth. A packet number is assigned to each packet (corresponding to its position and playback time) so that packets from multiple channels (paths) can be reassembled at the receiver. A start-up delay is set on the client side (in order of seconds) as a common practice in commercial streaming products. The client's buffer should host packets that arrive earlier than their playback times. The fraction of late packets, the probability that a packet is late, is used as a performance metric. We used the scheme proposed by Rejaie and Ortega [13] to allocate packets over multiple channels dynamically according to their bitrates and to allocate the clusters with a higher bitrate to the channels with higher throughput. The generated content packets at the server side are placed into a queue with priority given to the packets with an earlier 
playback time. The generated packets are transmitted and stored in the client-side buffer, and then the client starts to play the packets in its buffer at a constant rate. As confirmed by Wang et al. [14], out-of-order packets have a negligible effect on the fraction of late packets.

The distortion optimization problem is formulated as follows: the number of frames in a substream is $Y_{i}=$ $\left(t_{\text {busy }}+t_{\text {idle }}\right) / t_{\text {frame_duration. To find the optimum frame bitrate, }}$ the server makes a group of consecutive segment images into substreams (all images belong to scenes in the same cluster) and performs rate-distortion optimization. Normally, distortion is caused by source compression and packet loss. The compression distortion depends on the rate of the video stream and parameters of the distortion model, which depend on the encoded video sequence and encoding structure [15]. The total distortion for channel $j$, allocated to a cluster, is

$$
\begin{aligned}
D_{\text {packet_loss }} & =\alpha\left(\bar{L}_{T}+(1\right. \\
& \left.\left.-\bar{L}_{T} \exp \left\{-\frac{\operatorname{Tr}_{\text {delay }}}{\sum_{l=1}^{L}\left((\mathrm{LBR}-\Xi) \cdot \bar{P}_{l} \cdot a / r_{l}^{\text {out }}\right)}\right\}\right)\right),
\end{aligned}
$$

where $\alpha$ depends on the parameters related to the compression, $\bar{L}_{T}$ is average packet loss because of transmission error, $\operatorname{Tr}_{\text {delay }}$ is delay threshold, LBR is link balance rate, $\Xi$ is an overprovisioning factor, and $a$ is a parameter indicating whether link $l$ is being used for transmission. Suppose that $z=\{1, \ldots, Z\}$ is the cluster to be transmitted with bitrate $B_{s}^{z}, N_{c}$ is the number of alternative channels available to the cluster, $D_{s}^{z}$ is the source distortion of cluster $z$, and LBR is the fraction of link input and link output [15]. Hence, the distortion optimization problem is defined as

$$
\begin{array}{ll}
\operatorname{minimize} & D \\
& =\sum_{z=1}^{Z} \sum_{j=1}^{N_{c}}\left(\frac{\theta}{B_{s}-B_{s}^{z}}+D_{s}^{z}+D_{\text {packet_loss }}\right) \\
\text { subject to: } & \sum_{c} W_{c} \leq \sum_{z=1}^{Z} \sum_{j=1}^{N_{c}} F_{z j} B_{s} \\
& \sum_{j=1}^{N_{c}} F_{z j}=1 \\
& F_{s j} \geq 0, \quad N_{c} \geq 1, B_{s} \geq 0,
\end{array}
$$

where the first (15) constraint indicates that the link output should be less than or equal to the link input. We used convex programming formulation to simplify the goal function that is constructing a trade-off between allocation of the available channels to the segments and video distortion.

Finally, we define an optimization problem to choose the optimal segment size. Assume that segment $S$ has $X$ substreams. $Y_{x}$ is the number of frames in a substream. $W_{c}$ is the maximum available bandwidth of the channel in a short or long time slot, and $B_{x}=\left\{B_{1}, B_{2}, \ldots, B_{X}\right\}$ is the number of bits allocated to each frame in each substream $x\left(B_{\max }\right.$ is the maximum allowed bitrate). $\psi_{B}$ is the overall quality of the segment with the current bitrate. $\psi_{x, i}(B)$ is the quality (PSNR) of frame $y$ in substream $x$ with $B$ bits. Hence, our optimization problem is defined as the following to determine the best bitrate for each segment by maximizing the sum of the logarithms of the frame rates so that the average quality of the delivered video is maximized:

$$
\begin{array}{ll}
\text { maximize } & \psi_{B}=\sum_{x=1}^{X} \frac{1}{Y_{x}} \sum_{i=1}^{Y_{x}} \log \left(\psi_{x, i}\left(B_{x}\right)\right) \\
\text { subject to: } & \sum_{i=1}^{x} Y_{i} B_{i} \leq B_{\max } \\
& \sum_{i=1}^{x} Y_{i} B_{i} \leq W_{c} \\
& P_{\text {miss }}\left(W_{c}-W_{\text {miss }}\right) \leq \Delta W \\
& \rho_{s, l} \in\{0,1\}, \quad \forall s, l \\
& \sum_{s=1}^{S} \rho_{s, l}=1, \quad \forall l,
\end{array}
$$

where the third constraint in (21) is to take care of the maximum rate loss that licensees can tolerate, and the last two constraints are to ensure that each primary subcarrier is assigned to only one substream. The formulated optimization problem is nonlinear integer programming (NIP). We used the proposed algorithms in [1] to solve the problem, that is, the sequential fixing (SF) algorithm based on a linear relaxation and the greedy algorithm with proven optimality gap.

\section{Simulation Results}

In this section, we present the effectiveness evaluation of the proposed method using extensive simulations. To simulate video applications, we used Blue_Planet video sequence represented as a video with dramatic levels of motion activity. The video is in the common intermediate format (CIF, $352 \times$ 288). It has 5383 frames with a rate of $30 \mathrm{fps}$, and $336 \mathrm{GoPs}$ with GoP pattern of G16B3. The duration of a time slot is 0.03 seconds. First, we clustered the content features based on scene complexity and PSNR.

The hit rate for assigning each data point to its correct cluster is defined as the number of data points assigned to their original clusters over the total number of feature points. As can be seen in Figure 1, the data points are grouped in five clusters with a hit accuracy of more than $90 \%$. The salient advantage of the proposed method can be seen from the figure. With considerable difference in bitrate among the clusters, traditional fixed channel allocation regardless of the video scenes' bitrate results in wastage of precious network resources. However, in our method, the channel allocation is based on the video bitrate and the quality of the available channels: low-quality channels are assigned to lowbitrate clusters, and high-quality channels are reserved for 


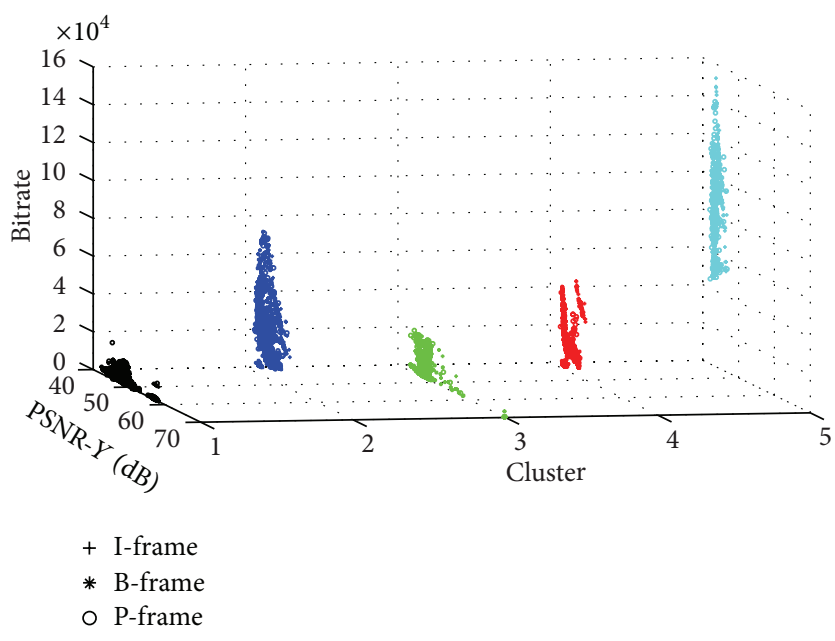

FIgURE 1: Clustering of the content.

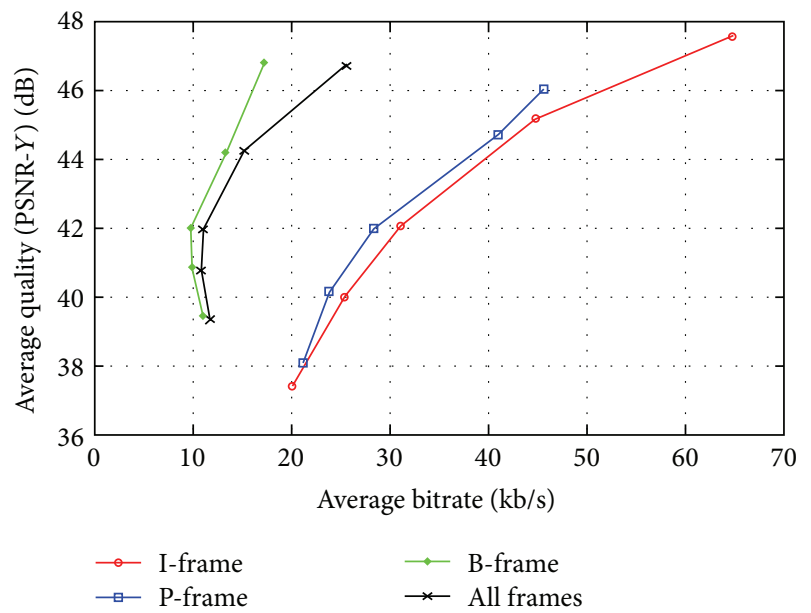

Figure 2: Rate-distortion for Blue_Planet video sequence.

the clusters with a higher bitrate. As discussed previously, we consider underlay mode for transmission of clusters with a lower bitrate to prevent transmission interruption.

Rate-distortion is used to characterize the compression efficiency of a video codec. Rate-distortion shows the quality of the compressed video measured in PSNR as a function of the video bitrate. Figure 2 shows rate-distortion for Blue_Planet video sequence in CIF format encoded in H.264/SVC for different frames. The lower the compressed bitrate, the more rate-distortion efficient the compression. The rate-distortion results in Figure 2 illustrate the general encoder characteristics verified for large sets of video test sequences. H.264/SVC compared to H.264/AVC and MPEG-4 gives salient efficiency in rate-distortion. For many encoding strategies, H.264/SVC yields almost two times the compression ratio for the equal video PSNR values.

Second, we need to evaluate the quality of the available channels and find the most appropriate channel for each cluster. We used real wireless traces from a $3 \mathrm{G}$ network including Game, UDP, P2P, and VoIP [16]. To achieve higher accuracy, we set the hyperparameters for various numbers of

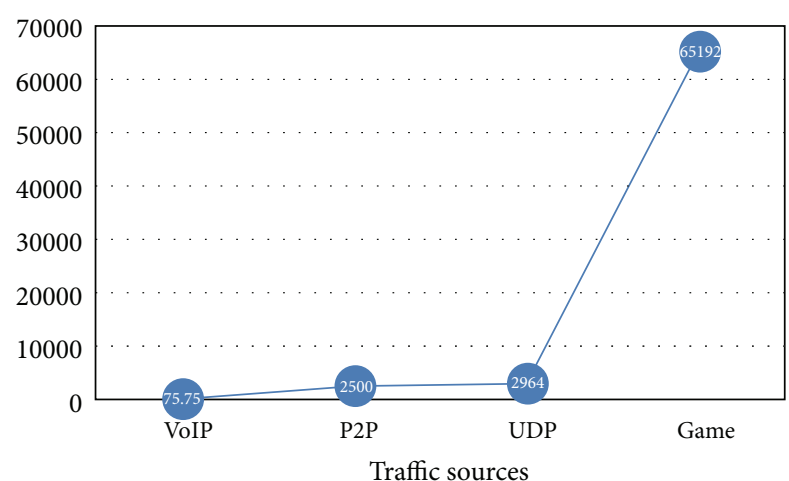

(a)

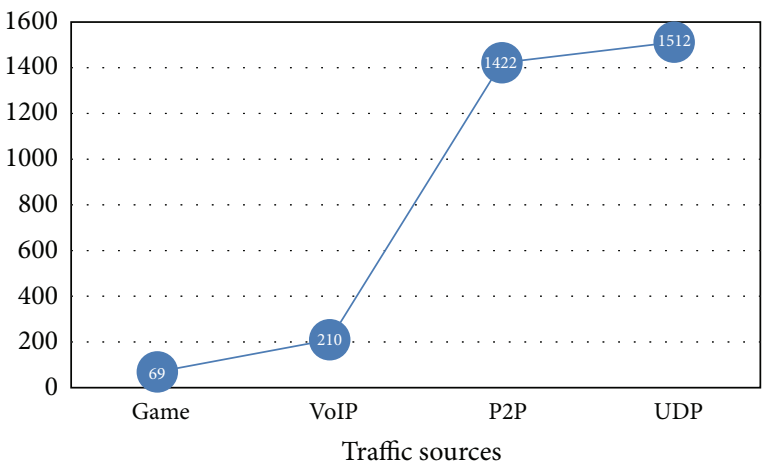

(b)

FIGURE 3: Average packet interarrival time (a) and average packet length (b).

traffic patterns. Figures 3(a) and 3(b) show the average packet interarrival time and the average packet length of the traffic sources, respectively.

Figure 3(a) demonstrates that packet interarrival time varies based on the requirements of various applications. VoIP obviously has a smaller interarrival time to prevent jitter effects; game applications have higher interarrival time than the other applications. In the case of packet length (Figure 3(b)), VoIP uses a smaller packet size again to minimize jitter; the UDP packet size is longer, whereas the game packet length varies based on game dynamics.

Because our clustering technique is nonparametric, it can cluster an unlimited number of traffic sources. For various numbers of traffic data points, the hyperparameters are set to achieve better accuracy. Figures 4(a), 4(b), and 5 show cluster parameter estimation results, that is, interarrival time scale and shape parameters, and idle time distribution for short and long time slots, for traffic sources from nonparametric variational inference [17].

The idle time distribution of the PU follows the Pareto heavy tail, as shown in Figure 5. From Figures 4 and 5, it is apparent that the parameters of the distribution method differ for each traffic source. Thereby, an appropriate channel is mapped to each content cluster according to its requirements.

The performance metric of the proposed method is video delivery quality [18], PSNR, which refers to a video's successful transmission time normalized by the PU idle-busy 


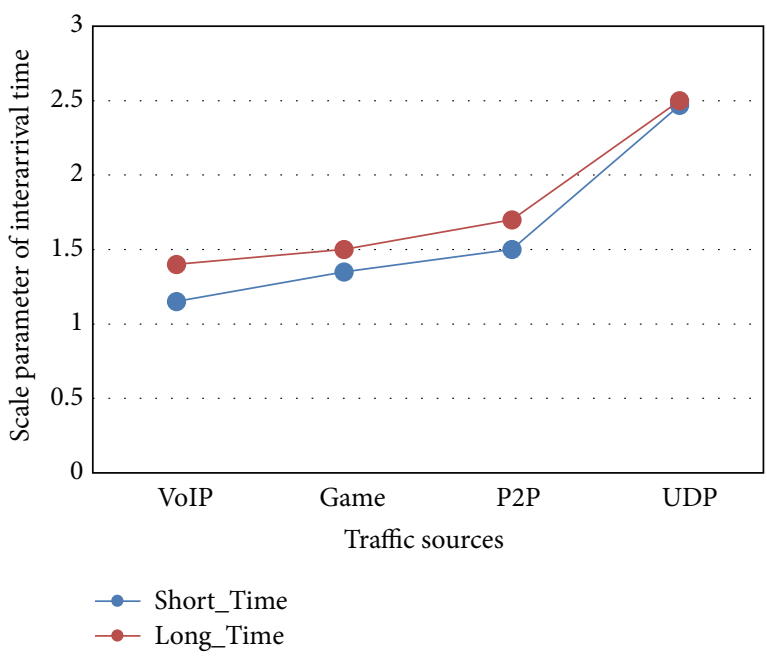

(a)

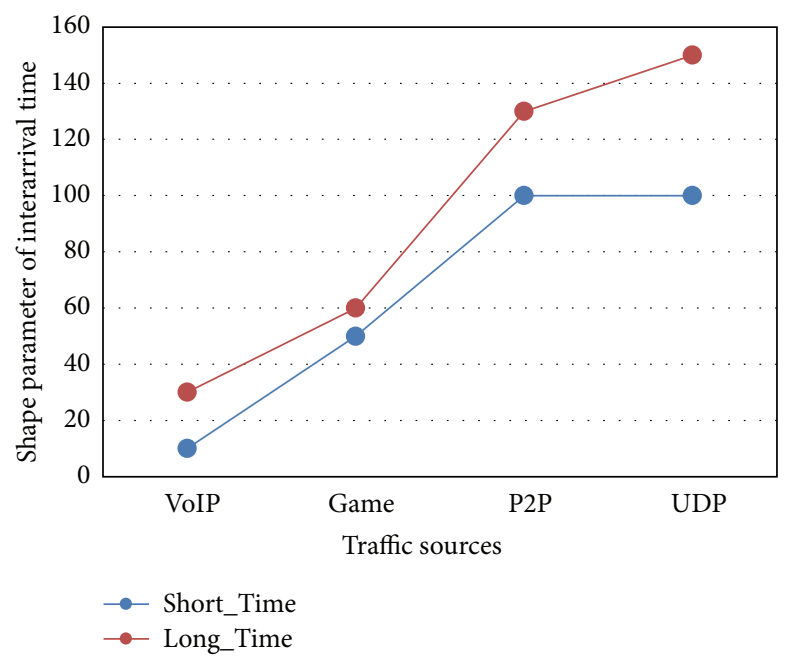

(b)

FIGURE 4: $\zeta^{C}$ (a) and $\zeta^{H}(\mathrm{~b})$ of packet interarrival time of different traffic patterns.

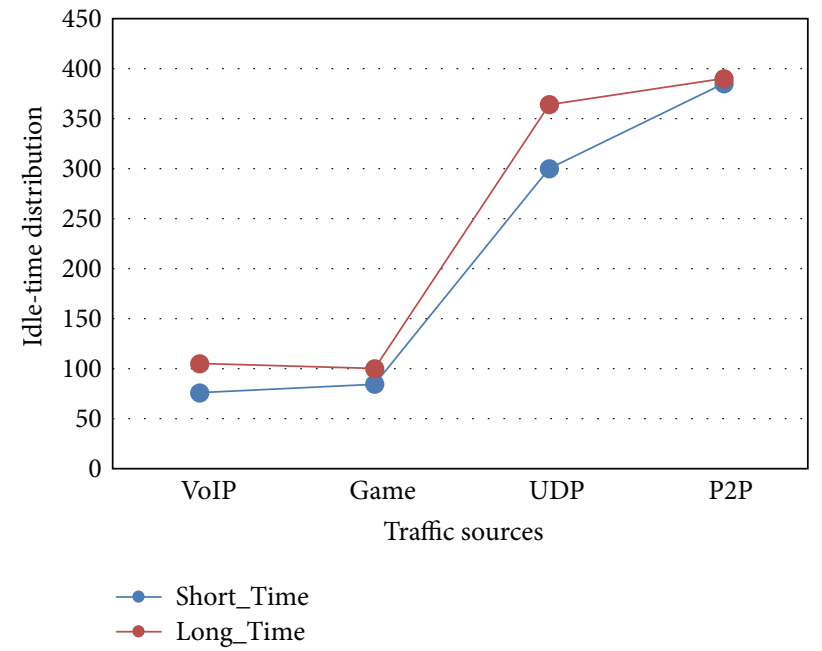

Figure 5: Idle time of both short and long time slots for different traffic patterns.

cycle. We assumed that channel utilization is 0.6 and that five pairs of probability of false and miss detection are $\{(0.1,0.45),(0.2,0.34),(0.3,0.21),(0.7,0.1),(0.9,0.03)\}$. We compared the performance of the proposed method with equal channel allocation. From Figure 6, it is evident that the best video quality is achieved when the false alarm probability is between 0.2 and 0.3 ; the probabilities of false alarm and misdetection are correlated such that decreasing one will normally increase the other. When the probability of a false alarm is larger, the rate of spectrum wastage will be higher, which results in lower bandwidth for the content clusters and consequently lower video quality. When the probability of misdetection is larger, interference with PUs increases, and it must be minimized. When the probability of a false alarm is lower than 0.2 and probability of misdetection

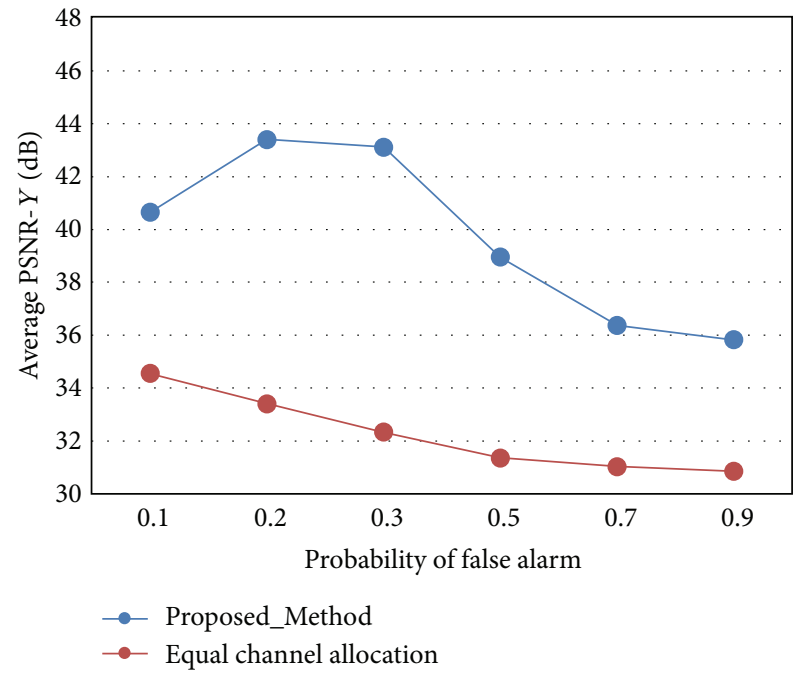

FIGURE 6: Average PSNR versus probability of false alarm.

is 0.34 , there will be interference between SUs, which will also result in degradation to video delivery quality.

\section{Conclusion}

In this paper, we studied primary spectrum utilization by SUs for video transmission. The sender clusters both the video content based on scene complexity and the traffic patterns based on a nonparametric technique using variational Bayesian inferences. Then, the content clusters are transmitted on multichannel CRNs according to the cluster requirements and the quality of the available channels. Underlay mode was discussed to switch the transmission at the time of PU arrival to avoid transmission interruption. Moreover, we defined a distortion optimization model based on the network's transmission mechanisms. We also optimized each 
segment's bitrate by maximizing the sum of the logarithms of the frame rate. From the experimental and simulation results, we have concluded that the proposed method has acceptable performance in terms of the quality of delivered video.

\section{Conflict of Interests}

The authors declare that there is no conflict of interests regarding the publication of this paper.

\section{Acknowledgment}

This research was funded by the MSIP (Ministry of Science, ICT \& Future Planning), Korea, in the ICT R\&D Program 2015.

\section{References}

[1] D. Hu and S. Mao, "Streaming scalable videos over multihop cognitive radio networks," IEEE Transactions on Wireless Communications, vol. 9, no. 11, pp. 3501-3511, 2010.

[2] Y. Ge, M. Chen, Y. Sun, Z. Li, Y. Wang, and E. Dutkiewicz, "QoS provisioning wireless multimedia transmission over cognitive radio networks," Multimedia Tools and Applications, vol. 67, no. 1, pp. 213-229, 2013.

[3] F. R. Yu, B. Sun, V. Krishnamurthy, and S. Ali, "Application layer QoS optimization for multimedia transmission over cognitive radio networks," Wireless Networks, vol. 17, no. 2, pp. 371-383, 2011.

[4] H. Luo, S. Ci, and D. Wu, "A cross-layer design for the performance improvement of real-time video transmission of secondary users over cognitive radio networks," IEEE Transactions on Circuits and Systems for Video Technology, vol. 21, no. 8, pp. 1040-1048, 2011.

[5] M. Thirunavukkarasu, M. Murugappan, and M. S. Mohan, "Multichannel cognitive cross layer optimization for improved video transmission," Journal of Computer Science, vol. 9, no. 1, pp. 43-54, 2013.

[6] H. T. Cheng and W. Zhuang, "Simple channel sensing order in cognitive radio networks," IEEE Journal on Selected Areas in Communications, vol. 29, no. 4, pp. 676-688, 2011.

[7] H. Qin and Y. Cui, "Cross-layer design of cognitive radio network for real time video streaming transmission," in Proceedings of the ISECS International Colloquium on Computing, Communication, Control, and Management (CCCM '09), vol. 3, pp. 376-379, IEEE, Sanya, China, August 2009.

[8] P. Wang, D. Niyato, and H. Jiang, "Voice-service capacity analysis for cognitive radio networks," IEEE Transactions on Vehicular Technology, vol. 59, no. 4, pp. 1779-1790, 2010.

[9] M. J. Piran, A. Ali, and D. Y. Suh, "Fuzzy-based sensor fusion for cognitive radio-based vehicular ad hoc and sensor networks," Mathematical Problems in Engineering, vol. 2015, Article ID 439272, 9 pages, 2015.

[10] M. J. Piran, Y. W. Cho, J. H. Yun, A. Ali, and D. Y. Suh, "Cognitive radio-based vehicular ad hoc and sensor networks," International Journal of Distributed Sensor Networks, vol. 2014, Article ID 154193, 11 pages, 2014.

[11] M. E. Ahmed, J. B. Song, Z. Han, and D. Y. Suh, "Sensingtransmission edifice using bayesian nonparametric traffic clustering in cognitive radio networks," IEEE Transactions on Mobile Computing, vol. 13, no. 9, pp. 2141-2155, 2014.
[12] V. Chakravarthy, X. Li, Z. Wu et al., "Novel overlay/underlay cognitive radio waveforms using SD-SMSE framework to enhance spectrum efficiency-part I: theoretical framework and analysis in AWGN channel," IEEE Transactions on Communications, vol. 57, no. 12, pp. 3794-3804, 2009.

[13] R. Rejaie and A. Ortega, "PALS: peer-to-peer adaptive layered streaming," in Proceedings of the 13th International Workshop on Network and Operating Systems Support for Digital Audio and Video, pp. 153-161, ACM, June 2003.

[14] B. Wang, W. Wei, Z. Guo, and D. Towsley, "Multipath live streaming via TCP: scheme, performance and benefits," $A C M$ Transactions on Multimedia Computing, Communications and Applications, vol. 5, no. 3, article 25, 2009.

[15] L. Zhou, X. Wang, W. Tu, G.-M. Muntean, and B. Geller, "Distributed scheduling scheme for video streaming over multichannel multi-radio multi-hop wireless networks," IEEE Journal on Selected Areas in Communications, vol. 28, no. 3, pp. 409-419, 2010.

[16] Data Base of a Community Resource for Archiving Wireless data at Dartmouth, http://crawdad.cs.dartmouth.edu/.

[17] K. Kurihara, M. Welling, and Y. W. Teh, "Collapsed variational dirichlet process mixture models," in Proceedings of the 20th International Joint Conference on Artificial Intelligence, 2007.

[18] C.-H. Ke, "myEvalSVC: an integrated simulation framework for evaluation of H.264/SVC transmission," KSII Transactions on Internet and Information Systems, vol. 6, no. 1, pp. 378-393, 2012. 

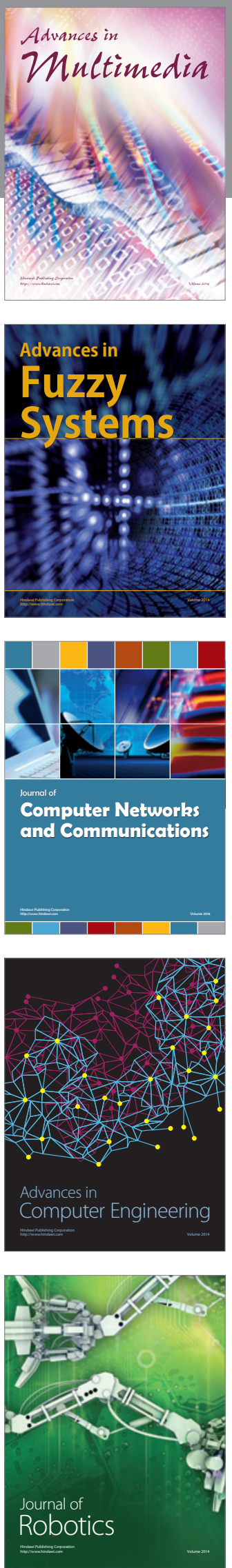

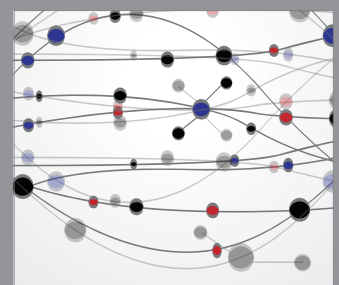

The Scientific World Journal
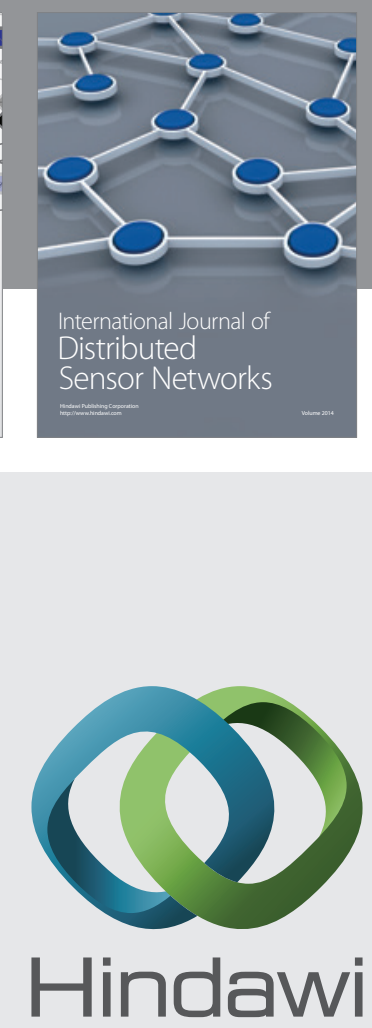

Submit your manuscripts at

http://www.hindawi.com
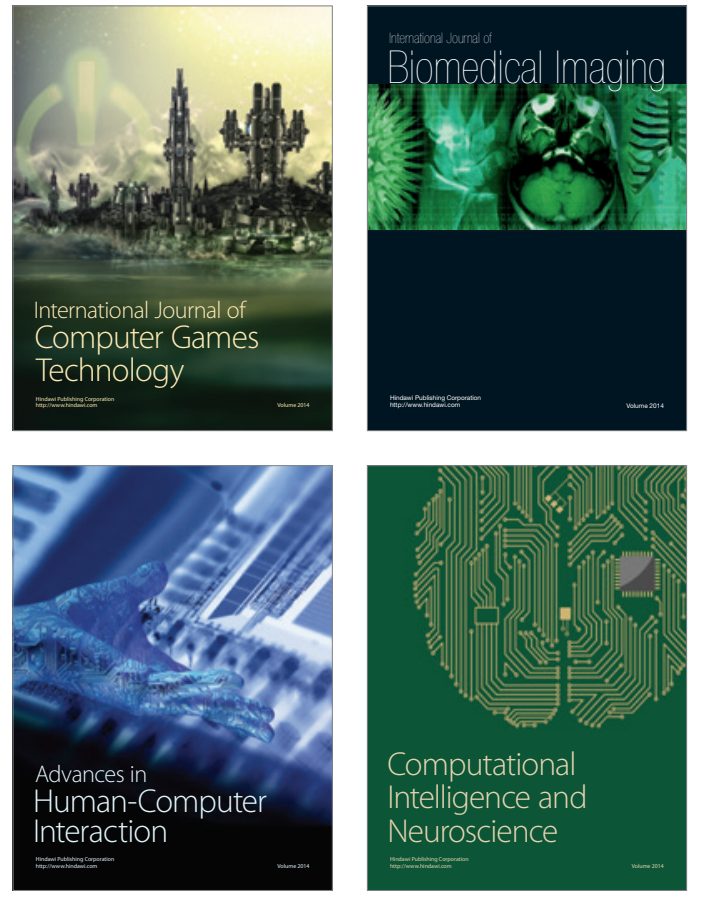
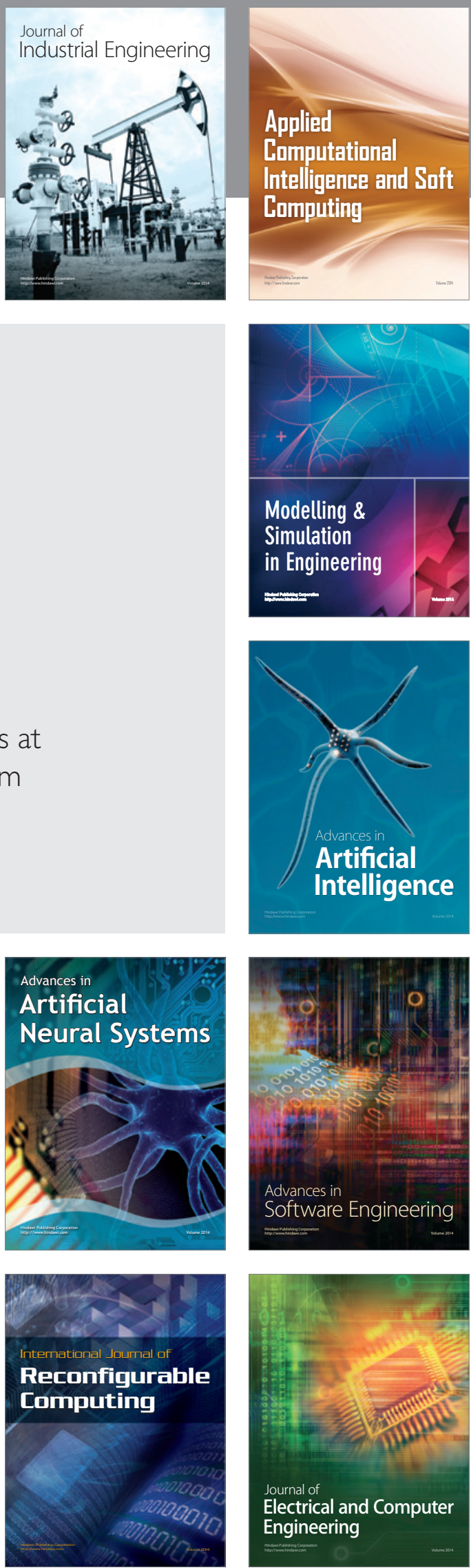